\title{
Self-force approach for radiation reaction
}

\author{
Lior M. Burko \\ Theoretical Astrophysics, California Institute of Technology, Pasadena, California 91125
}

\begin{abstract}
We overview the recently proposed mode-sum regularization prescription (MSRP) for the calculation of the local radiation-reaction forces, which are crucial for the orbital evolution of binaries. We then describe some new results which were obtained using MSRP, and discuss their importance for gravitational-wave astronomy.
\end{abstract}

The problem of including the radiation-reaction (RR) forces in the orbital evolution of a binary is a long-standing open problem. This problem is as yet unresolved even in the extreme mass-ratio limit, with the particle orbiting a non-rotating black hole, although there has been a remarkable progress obtained from various directions [1]. The conventional approach is to consider the fields in the far zone, and then use a balance argument to relate the far-zone fields to the local properties of the particle. The generic failure of such approaches [2] prompted the idea to calculate the local forces acting on the particle, including the RR forces. In the following we discuss the RR forces acting on a scalar point-like charge, but for electric or gravitational charges the basic ideas are similar. The $\mathrm{RR}$ force ${ }^{\mathrm{RR}} F^{\mu}$ which acts on a point-like scalar charge $q$ is given by [3]

$$
\mathrm{RR}^{\mu}(\tau)=q^{2}\left[\frac{1}{3}\left(\ddot{u}^{\mu}-u^{\mu} \dot{u}_{\nu} \dot{u}^{\nu}\right)+\frac{1}{6} \mathcal{R}^{\mu}+\int_{-\infty}^{\tau} \nabla^{\mu} G_{\mathrm{R}} d \tau^{\prime}\right],
$$

where $\mathcal{R}^{\mu}=R_{\nu}^{\mu} u^{\nu}+R_{\nu \sigma} u^{\nu} u^{\sigma} u^{\mu}-\frac{1}{2} R_{\nu}^{\nu} u^{\mu}, R_{\nu \sigma}$ is the Ricci tensor, $u^{\mu}$ is the charge's 4-velocity, a dot denotes (covariant) derivative with respect to proper time $\tau, \nabla_{\mu}$ denotes covariant differentiation, and $G_{\mathrm{R}}$ is the retarded Green's function. The first term is a local Abraham-Lorentz-Dirac type damping force, the second is a local force, which couples to Ricci-curvature and preserves conformal invariance, and the third is the so-called "tail" term, which arises from the failure of the Huygens principle in curved spacetime. The greatest problems in the calculation of the RR forces lurk in the tail term, because it requires the knowledge of $G_{\mathrm{R}}$ along the entire past world line of the charge. In addition, the self field of any particle diverges at the position of the particle, and the calculation of the $\mathrm{RR}$ forces will have to handle the infinities connected with the self field by providing a regularization prescription.

Recently, Ori proposed to approach the RR problem via mode decomposition [4]. Ori observed, that the individual Fourier-harmonic modes of the self field are

CP523, Gravitational Waves: Third Edoardo Amaldi Conference, edited by S. Meshkov (1) 2000 American Institute of Physics 1-56396-944-0/00/\$17.00 
bounded, also for a point-like particle, although the sum over all modes diverges. This observation is very useful, because the calculation of the individual modes is relatively easy. This still leaves the second, harder problem of having a regularization prescription to handle the mode sum. Very recently, Ori suggested MSRP [5], which is very successful for the few simple cases to which it has already been applied. In what follows, we overview MSRP very briefly, and describe some of the recent results which were obtained using it.

The tail part of the RR force can be decomposed into stationary Teukolsky modes, and then summed over the frequencies $\omega$ and the azymuthal numbers $m$. This force equals then the limit $\epsilon \rightarrow 0^{-}$of the sum over all $\ell$ modes, of the difference between the force sourced by the entire world line (the bare force ${ }^{\text {bare }} F_{\mu}^{\ell}$ ) and the force sourced by the half-infinite world line to the future of $\epsilon$, where the particle has proper time $\tau=0$, and $\epsilon$ is an event along the past $(\tau<0)$ world line. Next, we seek a function $h_{\mu}^{\ell}$ which is independent of $\epsilon$, such that the series $\sum_{\ell}\left({ }^{\text {bare }} F_{\mu}^{\ell}-h_{\mu}^{\ell}\right)$ converges. Once such a function is found, the regularized self force is then given by ${ }^{\text {tail }} F_{\mu}=\sum_{\ell}$ ( $\left.{ }^{\text {bare }} F_{\mu}^{\ell}-h_{\mu}^{\ell}\right)-d_{\mu}$, where $d_{\mu}$ is a finite valued function. MSRP [5] then shows, from a local integration of $G_{\mathrm{R}}$, that $h_{\mu}^{\ell}=a_{\mu} \ell+b_{\mu}+c_{\mu} \ell^{-1}$. MSRP also provides an algorithm for the calculation of the functions $a_{\mu}, b_{\mu}, c_{\mu}$ and $d_{\mu}$ analytically. It has been conjectured, that for all orbits $a_{\mu}=0=c_{\mu}$. (It was found to be true for all the special cases calculated so far.) If this is indeed the case, the tail force is given by

$$
{ }^{\text {tail }} F_{\mu}=\sum_{\ell}\left({ }^{\text {bare }} F_{\mu}^{\ell}-b_{\mu}\right)-d_{\mu} .
$$

Note that $b_{\mu}$ is just the limit bare $F_{\mu}^{\ell \rightarrow \infty}$, and that bare $F_{\mu}^{\ell}$ can be computed using the Teukolsky formalism. Alternatively, $b_{\mu}$ can also be calculated analytically using MSRP. The only remaining problem then, is to calculate $d_{\mu}$. Even though there is an algorithmic way to calculate $d_{\mu}$, this calculation is by no means easy. Although MSRP has been developed as yet only for very simplified cases, the approach is likely to be susceptible of generalization also for more realistic cases. If robust, MSRP can be of the greatest importance for the calculation of templates for gravitational-wave detection.

Table 1 displays the values of the MSRP parameters for the cases which have already been calculated. Note that the motion is not necessarily geodesic. The data in Table 1 may suggest the conjecture that $d_{\mu}$ exactly equals the sum of the two local terms of Eq. (1) (or its analog for other charge types). If this hypothesis is proved to hold in general, then a truely remarkable thing happens: the full RR force can be calculated directly from Eq. (2) when $d_{\mu}$ is ignored. It should be emphasized that presently the support for this far-reaching hypothesis is only the special cases listed in Table 1.

Next, we present some results which were obtained by application of this new approach. For the case of a static, minimally-coupled, massless scalar charge in Schwarzschild, the self force is known to equal zero [11]. For a static electric charge $q$ in Schwarzschild the self force is known to be purely radial and to be given by 
TABLE 1. Values of the MSRP parameters. All spacetimes are spherically symmetric. For all the cases $a_{\mu}=0=c_{\mu}$. The charge's spin is $s . \mathrm{A} *$ denotes the cases for which $b_{\mu}$ and $d_{\mu}$ were inferred indirectly, and a $\dagger$ denotes cases where the values of $b_{\mu}$ were corroborated numerically. Here, $I_{a}={ }_{2} F_{1}\left(1 / 2,1 / 2 ; 1 ; v^{2}\right)$ and $I_{b}={ }_{2} F_{1}\left(1 / 2,3 / 2 ; 1 ; v^{2}\right), v^{2}=-(d \varphi / d t)^{2} r^{2} / g_{t t}, a^{\mu}$ is the 4-acceleration, $a^{2}=\dot{u}_{\alpha} \dot{u}^{\alpha}$, and $k_{\mu}=\frac{q^{2}}{3}\left(\ddot{u}_{\mu}-u_{\mu} a^{2}\right)$.

\begin{tabular}{llllcc}
\hline Type of orbit & Spacetime & $s$ & $\mu$ & $b_{\mu} /\left[-q^{2} /\left(2 r^{2}\right)\right]$ & $d_{\mu}$ \\
\hline Static [5,6] & Minkowski & 0,1 & $t$ & 0 & 0 \\
Static [5,6] & Minkowski & 0,1 & $r$ & 1 & 0 \\
Static $^{\dagger}[5,7]$ & Schwarzschild & 0 & $r$ & $(r-M) /(r-2 M)$ & 0 \\
Circular $^{\dagger}[5,6]$ & Minkowski & 0 & $r$ & $\left(2 I_{a}-I_{b}\right) / u^{t}$ & 0 \\
Circular*† $^{*}[5,6]$ & Minkowski & 0 & $t$ & 0 & $k_{t}$ \\
Circular $^{* \dagger}[8]$ & Minkowski & 1 & $t$ & 0 & $2 k_{t}$ \\
Circular $^{\dagger}[5,9]$ & Schwarzschild & 0 & $r$ & $\left(2 I_{a}-\frac{r-3 M}{r-2 M} I_{b}\right) /\left(u^{t} \sqrt{-g_{t t}}\right)$ & 0 \\
Radial [10] & Minkowski & 0 & $r$ & $1-\dot{r}+r \ddot{r}$ & $k_{r}$ \\
Radial [10] & $g_{t t}(r) g_{r r}(r)=-1$ & 0 & $r$ & $1-\dot{r} u_{r}+r a_{r}$ & $k_{r}+q^{2} \mathcal{R}_{r} / 6$ \\
Radial [10] & $g_{t t}(r) g_{r r}(r)=-1$ & 0 & $t$ & $-\dot{r} u_{t}+r a_{t}$ & $k_{t}+q^{2} \mathcal{R}_{t} / 6$ \\
Static $^{*}[6]$ & Reissner-Nordström & 1 & $t$ & 0 & $q^{2} \mathcal{R}_{t} / 3$ \\
\hline
\end{tabular}

$f_{r}=q^{2} M r^{-3}(1-2 M / r)^{-1 / 2}[12]$. These results were recovered using MSRP in [7]. Note that for these two simple static cases the solution for the modes can be obtained analytically. In general, however, this is not expected to be possible, and the solution can be obtained only numerically.

The case of a scalar charge $q$ in uniform circular orbit around a Schwarzschild black hole was recently considered in [9]. The RR force was calculated numerically without any simplifying assumptions, such as far field or slow motion, and the solution is fully relativistic. Both the temporal and the azimuthal, dissipative components and the radial, conservative component of the $\mathrm{RR}$ force were computed. Figure 1 displays the behavior of the radial component of the RR force for both geodesic and non-geodesic orbits. In the slow motion and far field limits the force is repulsive, and behaves like ${ }^{\mathrm{RR}} F_{r} \approx q^{2} M^{2} \Omega^{2} / r^{2}$. However, in strong fields the force grows faster, and for fast motion is changed from repulsive to attractive. This expression for the radial, conservative $R R$ force may be very important for the detection of gravitational waves, and also for gravitational-waves astronomy. The conservative radial force causes an additional precession of the periastron of the particle's orbit, and thus induces a change in the frequency and phase of the emitted radiation [13]. Although the radial self force has been obtained only for the simple case of a point-like scalar charge, the result indicates that one can expect a non-zero periastron precession also for a small mass. However, the magnitude of the effect will very reasonably depend on the type of the charge. A large-magnitude effect can cause the entire search algorithm to fail in the very detection of the signal (depending also on the size of the template library), and a small effect will introduce errors in the parameters of the observed binary, namely, the wave form would fit the template of a system with parameters different from the parameter of the actual binary. Note that the conservative force depends not only on the 

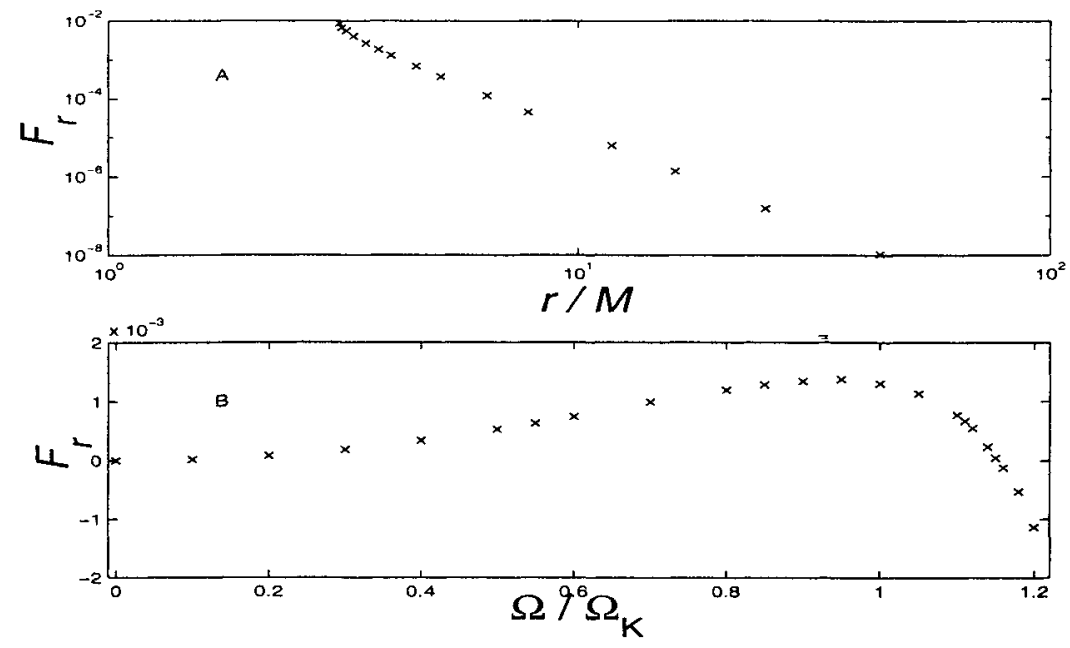

FIGURE 1. The radial RR force acting on a scalar charge in uniform circular orbit around a Schwarzschild black hole. Top panel (A): $F_{r}$ as a function of $r / M$ for geodesic orbits. Bottom panel (B): $F_{r}$ as a function of the angular velocity $\Omega$ in units of the Keplerian angular velocity $\Omega_{K}$, when the orbit is at $r=4 M$.

radiative modes of the field, but also on the non-radiative modes [13].

I thank Leor Barack and Amos Ori for discussions and for letting me use their results before their publication. This work was supported by NSF grants AST9731698 and PHY-9900776 and by NASA grant NAG5-6840.

\section{REFERENCES}

1. See, e.g., B. F. Schutz, Class. Quantum Grav. 16, A131 (1999).

2. S. A. Hughes, these proceedings; gr-qc/9910091.

3. T. C. Quinn and A. G. Wiseman, in preparation.

4. A. Ori, Phys. Lett. A202, 347 (1995); Phys. Rev. D55, 3444 (1997).

5. A. Ori, unpublished; For a brief outline of the proposed regularization method, see L. Barack and A. Ori, gr-qc/9911040.

6. L. M. Burko, unpublished.

7. L. M. Burko, Class. Quantum Grav. 17, 227 (2000).

8. L. M. Burko, Am. J. Phys. (in press), also gr-qc/9902079.

9. L. M. Burko, in preparation.

10. L. Barack and A. Ori, in preparation.

11. A. I. Zel'nikov and V. P. Frolov, Sov. Phys. JETP 55, 191 (1982); A. G. Wiseman, in preparation; A. E. Mayo, Phys. Rev. D 60, 104044 (1999).

12. A. G. Smith and C. M. Will, Phys. Rev. D 22, 1276 (1980).

13. L. M. Burko, in preparation. 